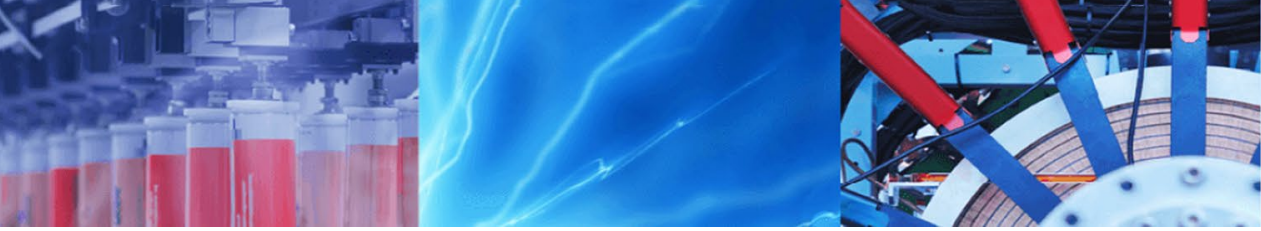

Research Article

\title{
Polymer/zeolite nano-composite hydrogels as promising water reservoir materials: effect of clinoptilolite content on physicochemical properties
}

\author{
Ali Olad ${ }^{1}$ (D) Negin Nouri ${ }^{1} \cdot$ Morad Eslamzadeh $^{1}$
}

(c) Springer Nature Switzerland AG 2019

\begin{abstract}
In this work, with the aim of improving the water uptake behavior and mechanical properties, a novel nano-composite hydrogel system was synthesized using clinoptilolite as a semi-layered zeolite. Accordingly, the gelatin/SCMC-g-poly (AA-CO-AAm)/clinoptilolite semi-IPN nano-composite hydrogel samples were synthesized and studied. The FTIR, XRD, SEM and EDX analysis were accomplished to confirm the successful synthesis of the nano-composite hydrogel samples. Additionally, to investigate the effect of clinoptilolite content on water uptake properties and gel characteristics of the synthesized nano-composite hydrogel samples, water absorption kinetics studies and rheological evaluations were carried out. It is obtained that, there is an optimum amount for additive content which can improve the hydrogel properties. Results showed that nano-composite hydrogel sample with $5 \% \mathrm{~W} / \mathrm{W}$ of clinoptilolite with respect to the gelatin amount has the best efficiency from the point of view of the water absorption properties and gel characteristics. This phenomenon is related to the creation of physical cross linking in the hydrogels' structure which leads to the creation of porous hydrogels.
\end{abstract}

Keywords Nano-composite hydrogel · Clinoptilolite · Semi-IPN · Water absorption · Gel characteristics

\section{Introduction}

Hydrogels are 3D cross linked polymer networks which have water retention behavior due to the presence of numerous hydrophilic functional groups in their structure [1-3]. These polymeric systems have various applications in the fields of agriculture and horticulture [4-6], wound dressings [7, 8], hygienic and cosmetic materials [9-11], water treatment [12] and medicine [13-15]. High hydrophilicity and high swelling ratio are two main factors for hydrogels which are achievable by graft polymerization of monomers on natural polymers such as gelatin, chitosan, alginate and cellulose [16]. According to the ineffective energy dissipation mechanisms and also due to the intrinsic structural heterogeneity in hydrogels, most of the synthetic hydrogels are brittle and mechanically weak [17].
To overcome this issue, scientists have designed various types of hydrogels which are mechanically stable. Interpenetrating network (IPN) hydrogels, semi-IPN hydrogels, double network (DN) hydrogels, and nano-composite (NC) hydrogels are some examples of the structurally modified hydrogels [18]. By combination of these methods, new types of hydrogels are achieved which have better properties than that of hydrogels have been made by each of the mentioned methods $[19,20]$. nano-composite hydrogels with semi-IPN structure, are a type of hydrogels which are more intended because of good mechanical properties besides their proper water absorption kinetics $[19,21]$. In semi-IPN hydrogels, one polymer chain is grafted and cross linked while the other polymer chain just interpenetrates the obtained network [22]. Various types of Nano-scaled materials such as clays, zeolites and

$\triangle$ Ali Olad, a.olad@yahoo.com | 1 Polymer Composites Research Laboratory, Department of Applied Chemistry, Faculty of Chemistry, University of Tabriz, Tabriz, Iran.

SN Applied Sciences (2019) 1:868 | https://doi.org/10.1007/s42452-019-0893-6

Received: 30 November 2018 / Accepted: 8 July 2019 / Published online: 18 July 2019 
carbon based materials are used for preparation of nanocomposite hydrogels [23]. Clinoptilolite which is a cheap and available zeolite with semi-layered structure and special properties, is mostly used for preparation of various polymeric nano-composites as well as super absorbent nano-composite hydrogels. This mineral additive has some advantages such as large surface area, low cost and high ion exchange capacity [24]. Application of layered or semi-layered Nano-materials into the hydrogel's structure causes to high porosity which is due to the physical cross linking of the polymer chains [25]. In this state, the polymer chains are surrounded by the Nano-layered particles in which the motion domain of the polymer chains is limited which leads to the stiffness [26]. The biocompatibility of the synthesized hydrogels is an important issue which is necessary to be considered. Accordingly, application of natural polymers such as gelatin, is very encouraged. Due to the weak physical and mechanical properties of the natural polymer based hydrogels, simultaneous application of natural and synthesized polymers in hydrogels' structure has been developed. Simultaneous application of natural and synthesized polymers in preparation of hydrogels, not only resolve the mechanical issues, but also it leads to achieve biocompatible hydrogel products $[27,28]$. So, synthesizing the hydrogels with semi-IPN structure, using both natural and synthetic polymers and by adding proper additives can lead to achieve a proper hydrogel system with desired properties.

In this study, a semi-IPN hydrogel was synthesized using gelatin and sulfated carboxymethyl cellulose as base natural polymers and poly(acrylic acid-co-acrylamide) as the grafting synthetic polymer. Afterwards, a neat hydrogel with optimized system was chosen based on the maximum water uptake capacity. To prepare desired nanocomposite hydrogel samples, certain amounts of clinoptilolite was added to the neat hydrogel formulation and then, the effect of clinoptilolite content on mechanical and water absorption properties of the nano-composite hydrogel was investigated. The Furrier-Transform Infrared spectroscopy (FTIR), X-ray Diffraction analysis (XRD) and Energy Dispersive X-ray analysis (EDX) were applied for structural studies. Also, Scanning Electron Microscopy (SEM) technique was accomplished to evaluate the morphological properties of the synthesized hydrogel samples. To investigate the water absorption properties of the neat and nano-composite hydrogel samples, water absorption kinetic studies were accomplished. Furthermore, for evaluating the effect of additive content on gel properties of the synthesized nano-composite hydrogel samples, the rheological studies were conducted. Based on the polymeric structure of the synthesized nano-composite hydrogel samples, and according to the bio compatibility and bio degradability of the used polymeric system and additives, the obtained polymer/zeolite nano-composite hydrogels have the potential of being used as water reservoir materials in agriculture and also they can be used as hygienic products.

\section{Experimental}

\subsection{Materials}

To synthesize gelatin/sulfated carboxymethyl cellulosegraft-Poly(acrylic acid-co-acrylamide)/clinoptilolite semiIPN nano-composite hydrogel samples, acrylic acid (AA) and acrylamide (AAm) monomers with the purity more than $95 \%$ were purchased from Merck Company (Germany). Also gelatin with commercial grade, was purchased from the Merck Company (Germany). The carboxymethyl cellulose sodium salt ( $\mathrm{NaCMC}$ ) with the purity of $>99.5 \%$ (viscosity of $1000-1500 \mathrm{mPa}$ for $4 \%$ aqueous solution at $25^{\circ} \mathrm{C}$ ) was purchased from the Fluka BioChemika (USA). $N, N^{\prime}$-methylene bisacrylamide (MBA) and ammonium persulfate (APS) were also purchased from Merck Company (Germany) as the cross linking agent and initiator, respectively. Additionally, $\mathrm{N}, \mathrm{N}$-dimethylformamide (DMF), chlorosulfunic acid were purchased from the Merck Company (Gremany) as active reagents for sulfating the CMC. Ethanol (96\%) were purchased from Razi Company (Iran, Arak). All the chemicals were used as received.

\subsection{Preparation of sulfated carboxymethyl cellulose}

To prepare sulfated carboxymethyl cellulose (SCMC), $5 \mathrm{~g}$ of CMC was added to a mixture of chlorosulfunic acid $(8 \mathrm{ml})$ and DMF $(40 \mathrm{ml})$ in a one necked flask equipped with a reflux column and then the mixture was stirred for $4 \mathrm{~h}$ at $60^{\circ} \mathrm{C}$. Afterwards, the $\mathrm{pH}$ value of the obtained brown colored solution was adjusted at 10-11 by an $8 \mathrm{M}$ $\mathrm{NaOH}$ aqueous solution then $50 \mathrm{ml}$ of ethanol was added to the mixture as the anti-solvent agent that in this state a pale brown sediment deposited in the bottom of the flask. Finally, the obtained solid was washed severally with distilled water and dried at $60^{\circ} \mathrm{C}$ in an oven.

\subsection{Designation and allocation of factors}

Taguchi method was used for preparation of neat hydrogel samples for investigating their swelling capacity and choose a sample with an optimum equilibrium water absorption capacity. Accordingly, different values of the main natural polymers, synthetic monomers, initiator and cross linker were inserted as effective parameters to the Taguchi software that a standard orthogonal array (L25) was achieved. Table 1 shows the variables and their 
Table 1 Different levels of each factor and their values

\begin{tabular}{lllllll}
\hline Factor & Symbol & Level 1 & Level 2 & Level 3 & Level 4 & Level 5 \\
\hline Gelatin (g) & Gel. & 1 & 2 & 4 & 8 & 16 \\
SCMC/gelatin (W/W \%) & SCMC/Gel. & 1 & 5 & 10 & 15 & 20 \\
AA (ml) & AA & 0.5 & 0.7 & 1 & 1.3 & 1.5 \\
AA/AAm (molar ratio \%) & AA/AAm & 10 & 15 & 20 & 25 & 30 \\
Initiator (g) & APS & 0.01 & 0.03 & 0.05 & 0.07 & 0.1 \\
Cross linker (mg) & MBA & 1 & 3 & 5 & 7 & 10 \\
\hline
\end{tabular}

different levels which were uploaded on Taguchi software. According to the synthesis process achieved from Taguchi software, 25 different neat hydrogel samples were synthesized and their equilibrium swelling amounts were calculated. Eventually, the obtained results were examined by Minitab 18 software using the statistical method for analysis of variance (ANOVA).

\subsection{Preparation of neat and nano-composite hydrogel samples}

To synthesize neat and nano-composite hydrogel samples, a free radical polymerization method was used in which the poly(acrylic acid-co-acrylamide) chains were grafted on SCMC and the obtained SCMC-graft-Poly(AA-CO-AAm) was cross linked by MBA while the gelatin was interpenetrating into the obtained network. For preparing neat hydrogel samples, first of all, certain amount of gelatin was dissolved in $30 \mathrm{ml}$ of distilled water in a round bottomed two necked flask at $45^{\circ} \mathrm{C}$ and then proper amount of $70 \%$ neutralized AA was added to the solution. After 10 min of stirring under nitrogen gas stream, according to the designed orthogonal array, certain amount of AAm was added to the solution and the solution was stirred vigorously to let the monomers to be dissolved completely. Afterwards, certain amount of MBA which was given in Taguchi design, was added to the solution and the solution was let to be mixed for half an hour under the nitrogen gas stream. Finally, the obtained homogeneous solution was poured into a plate and immediately the APS, solved in $5 \mathrm{ml}$ of distilled water, was added to the solution and then the plate was left in a fixed place under nitrogen atmosphere to let the hydrogel film to be formed. After $24 \mathrm{~h}$, the obtained hydrogel film was washed thoroughly with $96 \%$ ethanol and dried in an oven for $24 \mathrm{~h}$ at $60^{\circ} \mathrm{C}$. To remove the probable unreacted chemicals, the dried hydrogel film was immersed in fresh distilled water and the water was changed for three or four times during the immersion period. To synthesize nano-composite hydrogel samples, the synthesis process was the same with neat hydrogel but after dissolving the gelatin in distilled water, proper amounts of clinoptilolite were dispersed in the solution using an ultrasonic probe with the power of
$30 \mathrm{~kW}$ in a water-ice bath. For synthesizing nano-composite hydrogel samples, clinoptilolite was added in different amounts of $1 \%, 3 \%, 5 \%, 7 \%$ and $10 \%$ W/W with respect to the gelatin amount. The amount of the all chemicals was chosen based on the Taguchi design which is given in Table 2.

\subsection{Characterization}

To accomplish the structural analysis, Fourier-Transform Infrared (FTIR) spectroscopy and X-ray Diffraction (XRD) analysis were applied using Perkin Elmer 65 and Philips PW1730 instruments respectively. Also, Energy-dispersive $X$-ray (EDX) spectroscopy was done to investigate the elemental composition of the synthesized hydrogel samples using a MIRA3 TESCAN instrument. For morphological evaluation of the prepared hydrogel samples, Scanning Electron Microscopy (SEM) technique was accomplished using a MIRA3 TESCAN instrument. Rheological studies were carried out to confirm the effect of clinoptilolite addition on gel properties of the synthesized nano-composite hydrogel samples. For this aim, an Anton Paar MCR301 Rheometer equipped with a plate-plate geometry (plates diameter of $25 \mathrm{~mm}$ and gap of $1 \mathrm{~mm}$ ) was used at $25^{\circ} \mathrm{C}$.

\subsection{Water absorption properties}

\subsubsection{Equilibrium water absorption studies}

To investigate the water absorption ability of synthesized neat and nano-composite hydrogel samples, a standard method was used in which certain amount of each hydrogel sample was moved into a stainless steel grid container and the whole system was immersed in $100 \mathrm{ml}$ of de-ionized water at room temperature $\left(25^{\circ} \mathrm{C}\right)$. The system was moved out of the water at certain time intervals and after removing the excess surface water by a clean tissue paper, the swollen system was weighed. This process was continued until a stable water uptake amount was achieved. Knowing the weight of the grid container, the weight of the absorbed water can be easily obtained. The equilibrium water uptake amount at each time interval, was calculated using the Eq. (1): 
Table 2 Design of experiments (L25 orthogonal array)

\begin{tabular}{|c|c|c|c|c|c|c|}
\hline Test number & Gel. & SCMC/Gel. & AA & AA/AAm & APS & MBA \\
\hline 1 & Level 1 & Level 1 & Level 1 & Level 1 & Level 1 & Level 1 \\
\hline 2 & Level 1 & Level 2 & Level 2 & Level 2 & Level 2 & Level 2 \\
\hline 3 & Level 1 & Level 3 & Level 3 & Level 3 & Level 3 & Level 3 \\
\hline 4 & Level 1 & Level 4 & Level 4 & Level 4 & Level 4 & Level 4 \\
\hline 5 & Level 1 & Level 5 & Level 5 & Level 5 & Level 5 & Level 5 \\
\hline 6 & Level 2 & Level 1 & Level 2 & Level 3 & Level 4 & Level 5 \\
\hline 7 & Level 2 & Level 2 & Level 3 & Level 4 & Level 5 & Level 1 \\
\hline 8 & Level 2 & Level 3 & Level 4 & Level 5 & Level 1 & Level 2 \\
\hline 9 & Level 2 & Level 4 & Level 5 & Level 1 & Level 2 & Level 3 \\
\hline 10 & Level 2 & Level 5 & Level 1 & Level 2 & Level 3 & Level 4 \\
\hline 11 & Level 3 & Level 1 & Level 3 & Level 5 & Level 2 & Level 4 \\
\hline 12 & Level 3 & Level 2 & Level 4 & Level 1 & Level 3 & Level 5 \\
\hline 13 & Level 3 & Level 3 & Level 5 & Level 2 & Level 4 & Level 1 \\
\hline 14 & Level 3 & Level 4 & Level 1 & Level 3 & Level 5 & Level 2 \\
\hline 15 & Level 3 & Level 5 & Level 2 & Level 4 & Level 1 & Level 3 \\
\hline 16 & Level 4 & Level 1 & Level 4 & Level 2 & Level 5 & Level 3 \\
\hline 17 & Level 4 & Level 2 & Level 5 & Level 3 & Level 1 & Level 4 \\
\hline 18 & Level 4 & Level 3 & Level 1 & Level 4 & Level 2 & Level 5 \\
\hline 19 & Level 4 & Level 4 & Level 2 & Level 5 & Level 3 & Level 1 \\
\hline 20 & Level 4 & Level 5 & Level 3 & Level 1 & Level 4 & Level 2 \\
\hline 21 & Level 5 & Level 1 & Level 5 & Level 4 & Level 3 & Level 2 \\
\hline 22 & Level 5 & Level 2 & Level 1 & Level 5 & Level 4 & Level 3 \\
\hline 23 & Level 5 & Level 3 & Level 2 & Level 1 & Level 5 & Level 4 \\
\hline 24 & Level 5 & Level 4 & Level 3 & Level 2 & Level 1 & Level 5 \\
\hline 25 & Level 5 & Level 5 & Level 4 & Level 3 & Level 2 & Level 1 \\
\hline
\end{tabular}

$k_{s}=\frac{1}{A W_{\infty}^{2}}$

In these formulae the $W_{t}$ and $W_{\infty}$ are in-time and final water absorption amounts, respectively. $B$ is the inverse maximum water uptake amount and $A$ is the inverse initial swelling rate which are obtained from the slope and the intercept of the $\mathrm{t} / \mathrm{W}$ versus time graph, respectively. The swelling rate constant of the hydrogels is defined by $k_{s}$ factor which shows the ability of a hydrogel to get swollen. For hydrogels with more inter-linked pores, the rate of solvent penetration into the hydrogel structure is low, so the $\mathrm{k}_{\mathrm{s}}$ factor for these kind of hydrogels is decreased.

\subsubsection{Water absorption mechanism}

$\frac{t}{W}=A+B t$

$B=\frac{1}{W_{\infty}}$

$A=\frac{1}{\left(\frac{d W}{d t}\right)_{0}}$

To investigate the water absorption mechanism in hydrogel samples, the $\ln (\mathrm{t})$ versus $\ln \mathrm{W}_{\mathrm{t}} / \mathrm{W}_{\infty}$ graph was illustrated for initial $60 \%$ of the total water uptake amount. The graph's equation is as the following:

$\frac{W_{t}}{W_{\infty}}=k t^{n}$ 
In Eq. (6), $W_{t}$ and $W_{\infty}$ are in-time and final water uptake amounts, respectively. " $k$ " is the specific relative constant and " $n$ " is the factor which shows the diffusion mechanism of the absorbed water. $k$ and $n$ are obtained from the intercept and slope of the graph, respectively. There are two main diffusion phenomena for viscoelastic materials such as hydrogels that include Fickian or non-Fickian diffusion mechanisms. According to the obtained values for " $n$ " factor, the Fickian diffusion mechanism is occurred when the " $n$ " factor value is less than $0.5(n<0.5)$ in which the rate of solvent diffusion is not equal to the polymer network relaxation rate $\left(R_{\text {rel. }} \ll R_{\text {diff. }}\right.$ or $\left.R_{\text {rel }} \gg R_{\text {diff. }}\right)$. On the other hand, for " $\mathrm{n}$ " factor values between 0.5 and $1(0.5<\mathrm{n}<1)$, the diffusion mechanism is non-Fickian that in this case the rate of solvent penetration is equal to the polymer network relaxation rate. For non-Fickian diffusion mechanisms, there are two other cases with " $n$ " factor values equal to $1(n=1)$ or more than $1(n>1)$ that in these cases the rate of diffusion is equal to the rate of polymer network relaxation but there is an extra surface entry resistance $[29,30]$. For " $n$ " factor values equal to 1 , the diffusion mechanism is called case-II diffusion mechanism while for " $n$ " factor values more than 1 , it is called super case-II diffusion mechanism.

\subsubsection{Water absorption rate study}

Another kinetic parameter which is considered in hydrogels is the water absorption rate constant which shows how fast a hydrogel can absorb the water. The more a hydrogel is porous, the more this constant is, because the contact area between the water and hydrogel sample is increased which leads to more interactions between water molecules and hydrophilic functional groups in hydrogel structure. By illustrating the $-\ln \left(1-\left(\mathrm{W}_{\mathrm{t}} / \mathrm{W}_{\infty}\right)\right)$ versus time graph the water absorption rate constant $\left(k_{w}\right)$ is obtained from the slope of the graph. The respective equation for this graph is as the following (Eq. 7):

$W_{t}=W_{\infty}\left(1-e^{-k_{w} t}\right)$

where $W_{t}$ and $W_{\infty}$ are in-time and final water uptake amounts, respectively. $\mathrm{k}_{\mathrm{w}}$ is the water absorption constant.

\subsection{Rheological studies}

As the clinoptilolite is a semi-layered zeolite so it can affect the gel properties of the synthesized hydrogels due to probable creation of physical cross links between the clinoptilolite and graft co-polymer chains. To investigate the gel properties of the synthesized nano-composite hydrogels, rheological assay was accomplished. Firstly, to determine the Linear Viscoelastic (LVE) region, a strain sweep test was applied to the hydrogel samples at a constant frequency of $\omega=10 \mathrm{~Hz}$. Additionally, to investigate the viscoelastic behavior of the hydrogel samples at a constant strain $(\gamma=0.5)$ the frequency sweep tests were assessed over a wide range of angular frequencies $(0.1-100 \mathrm{~Hz})$.

\section{Results and discussion}

\subsection{Morphological studies}

To investigate the effect of the addition of clinoptilolite on morphology of the synthesized nano-composite hydrogels and also to compare the porosity of the nano-composite hydrogel samples with the neat hydrogel sample, SEM technique was assessed. The images for neat and nanocomposite hydrogel samples in dry state were achieved after lyophilization of the samples. As it is seen in Fig. 1a, the neat hydrogel sample has a complete uniform and smooth surface with no efficient porosity which leads to low water uptake capacity. In contrast, as it is seen in Fig. $1 \mathrm{~b}$, the lyophilized nano-composite hydrogel sample with an optimum amount of clinoptilolite, has a wrinkled surface with uniform dispersion of clinoptilolite on it. Also, at the points in which the graft co-polymer chains have been physically cross linked by clinoptilolite, deep gaps and pores are observed. These gaps and holes leads to high water uptake capacity which is due to the increase of the contact area between water and nano-composite hydrogel sample. Also, for completely swollen nanocomposite hydrogel sample (Fig. 1C), it is observed that the pores are inter-linked which leads to good water uptake properties and slow release behavior. According to the obtained images using the SEM technique, it is concluded that the addition of clinoptilolite can affect the water uptake capacity by increasing the porosity of the nano-composite hydrogels compared to the neat hydrogel sample.

\subsection{EDX analysis}

To investigate the elemental composition of the synthesized nano-composite hydrogel samples, and also to confirm the incorporation of clinoptilolite in the nanocomposite hydrogels structure, EDX analysis was accomplished. As it is seen in Fig. 2, there are some peaks which are belonged to various elements. $\mathrm{C}, \mathrm{O}$ and $\mathrm{N}$ peaks are attributed to the polymeric network which is made of AA, AAm, SCMC and gelatin. The height of the peaks shows the quantity of each element in hydrogel samples. Additionally, presence of $\mathrm{S}$ peaks in this pattern confirms 

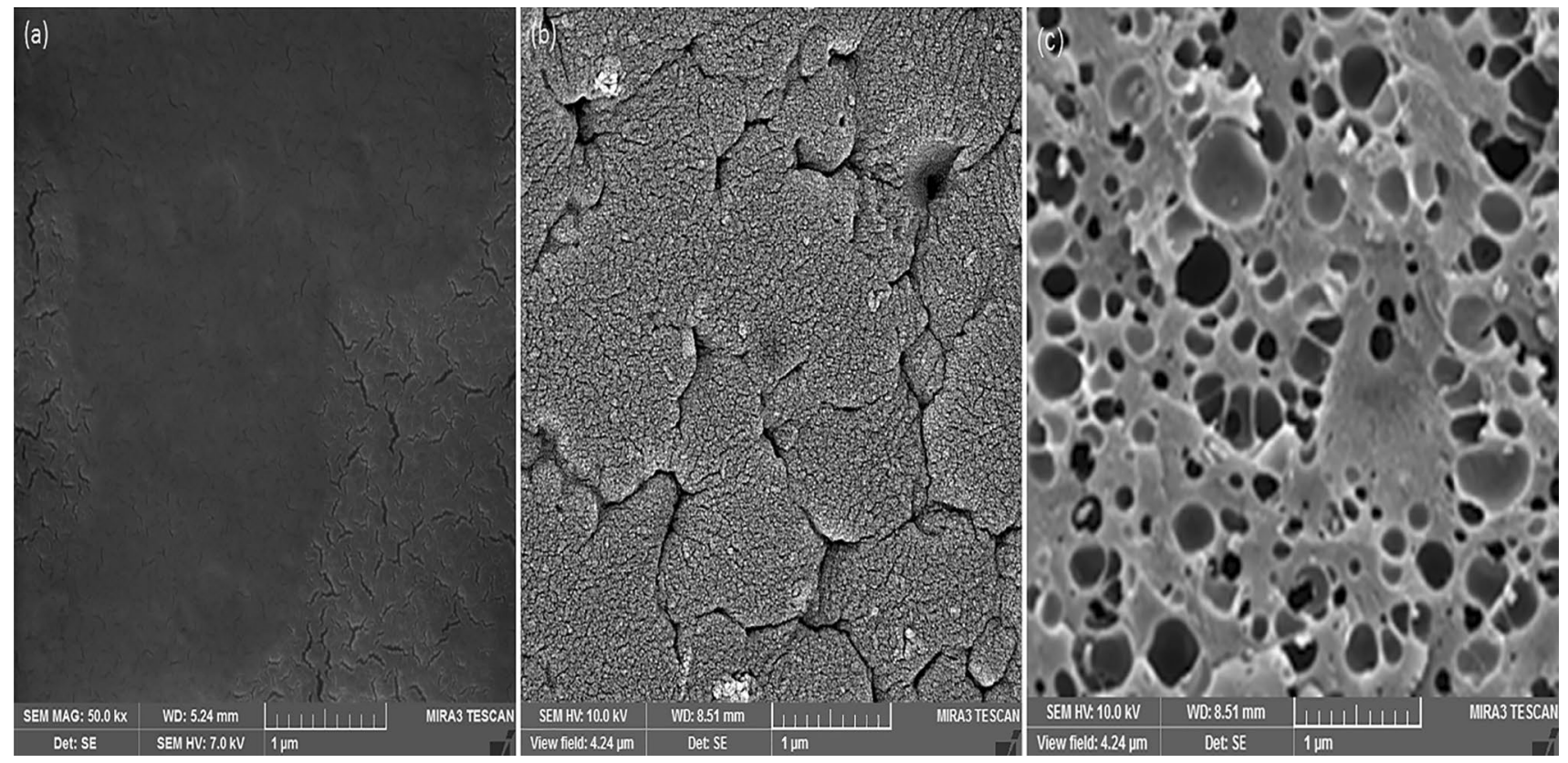

Fig. 1 SEM images of a neat hydrogel, b lyophilized nano-composite hydrogel and $\mathbf{c}$ swollen nano-composite hydrogel in equilibrium state at a same magnification

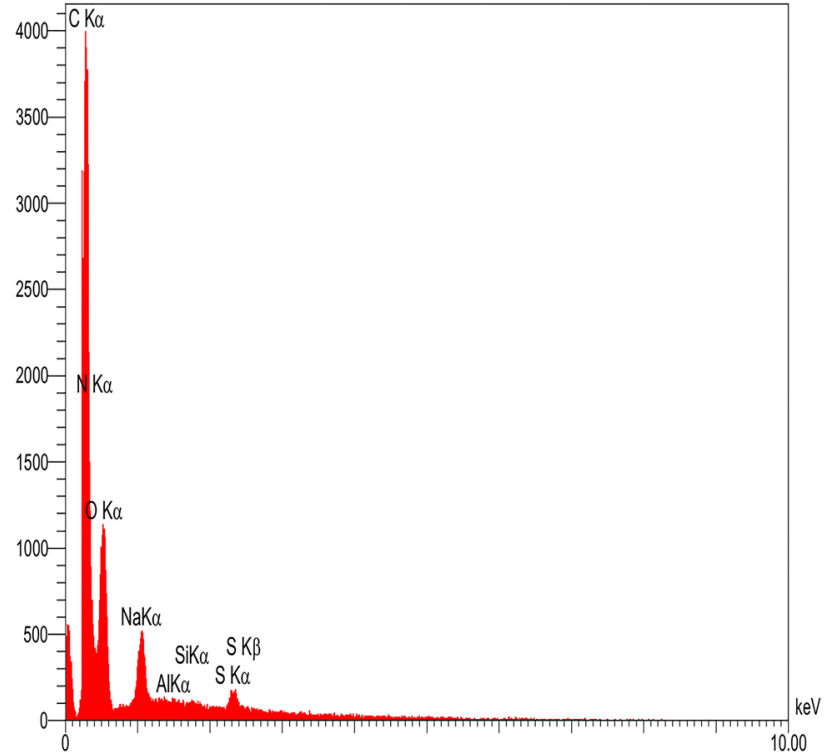

Fig. 2 EDX pattern for Gelatin/SCMC-graft-poly (AA-co-AAm)/ clinoptilolite nano-composite hydrogel sample

the incorporation of SCMC in hydrogels structure. Furthermore, presence of $\mathrm{Al}, \mathrm{Na}$ and Si peaks in this pattern confirms the successful synthesis of nano-composite hydrogels with clinoptilolite as an additive.

\subsection{FTIR studies}

To evaluate the structure of the synthesized neat and nano-composite hydrogel samples, FTIR analysis was done. In Fig. 3, the FTIR spectra of gelatin, clinoptilolite, CMC, SCMC, neat hydrogel and nano-composite hydrogel have been illustrated. As it is seen in gelatin FTIR spectrum, there is a wide sharp peak at $3435 \mathrm{~cm}^{-1}$ which is attributed to the stretching vibration of $\mathrm{O}-\mathrm{H}$ bonds which has overlapped with the stretching vibrations of $\mathrm{N}-\mathrm{H}$ bonds in amide $A$ groups at this region. There is also a shoulder at about $3070 \mathrm{~cm}^{-1}$ which is attributed to the stretching vibrations of amide $B$ groups in gelatin structure. The peak at $2947 \mathrm{~cm}^{-1}$ is related to the stretching vibrations of amide B C-H bonds. There are also amide I and amide II in gelatin structure that their belonged peaks are observed at $1636 \mathrm{~cm}^{-1}$ and $1540 \mathrm{~cm}^{-1}$ which are due to the stretching vibrations of $-\mathrm{C}=\mathrm{O}$ bonds in amide $\mathrm{I}$ and $\mathrm{N}-\mathrm{H}$ deformation in amide II, respectively [31]. Furthermore, a small peak at $1450 \mathrm{~cm}^{-1}$ with a shoulder at $1400 \mathrm{~cm}^{-1}$ is observed in FTIR spectrum of gelatin which is related to the deformation vibrations of $-\mathrm{C}-\mathrm{H}$ groups and the symmetrical stretching vibrations of $-\mathrm{C}=\mathrm{O}$ groups in gelatin [32]. As it is seen in Fig. 3, the FTIR spectrum of CMC has a broad absorption band at $3440 \mathrm{~cm}^{-1}$ which is attributed to the stretching vibrations of $\mathrm{O}-\mathrm{H}$ bonds. Also, peaks at $2917 \mathrm{~cm}^{-1}, 1635 \mathrm{~cm}^{-1}$ and $1037 \mathrm{~cm}^{-1}$ are related to the stretching vibrations of $\mathrm{C}-\mathrm{H}$ bonds in methylene groups, $-\mathrm{C}=\mathrm{O}$ bonds of carboxyl groups and $\beta-1,4$-glycosidic 


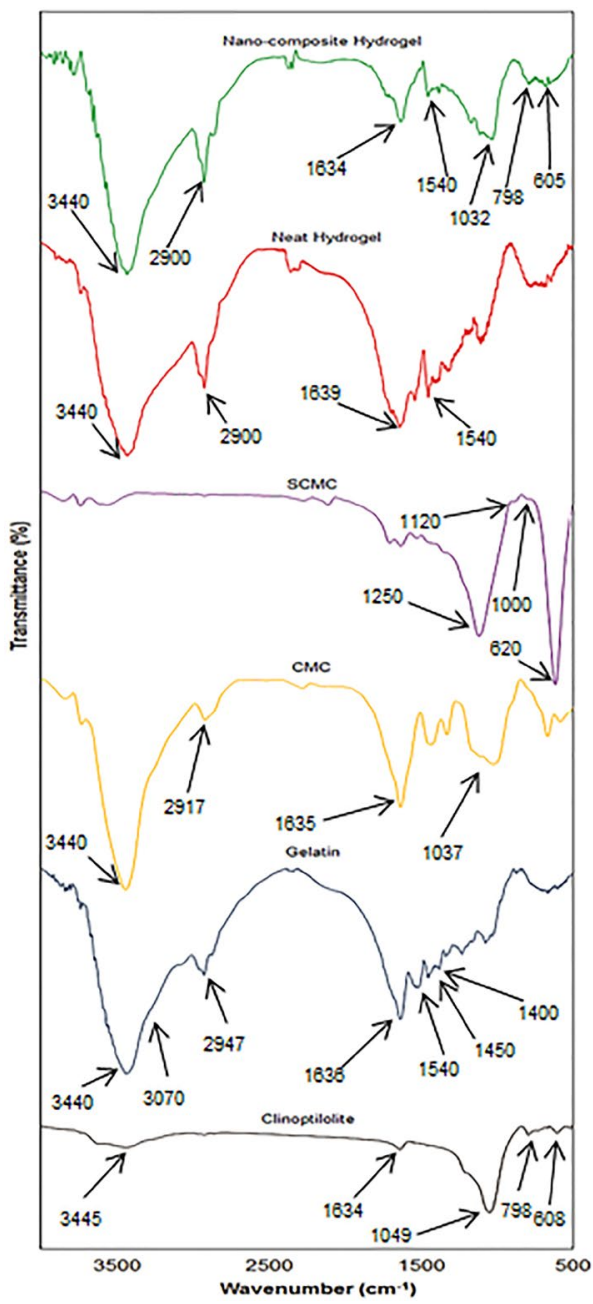

Fig. 3 FTIR spectra of the natural polymer, additive, neat hydrogel and nano-composite hydrogel

bonds in CMC structure, respectively [1]. As it is seen in FTIR spectrum of SCMC, absorbing bands at $1250 \mathrm{~cm}^{-1}$ and $620 \mathrm{~cm}^{-1}$ are attributed to the asymmetric stretching vibrations of $\mathrm{S}=\mathrm{O}$ functional groups. Additionally, there is a small peak at about $1000 \mathrm{~cm}^{-1}$ which is belonged to the symmetric stretching vibrations of $\mathrm{S}=\mathrm{O}$ groups. A strong absorbing band at $1120 \mathrm{~cm}^{-1}$ is attributed to the symmetric stretching vibrations of the $\mathrm{C}-\mathrm{O}-\mathrm{S}$ groups in SCMC structure [1]. As shown in Fig. 3, for clinoptilolite, a broad absorbing band is observed at $3445 \mathrm{~cm}^{-1}$ which is related to the stretching vibration of hydroxyl functional groups in $\mathrm{Al}-\mathrm{OH}-\mathrm{Al}$ and $\mathrm{Si}-\mathrm{OH}-\mathrm{Si}$ groups. Additionally, the peaks at $1634 \mathrm{~cm}^{-1}$ and $1049 \mathrm{~cm}^{-1}$ are attributed to $\mathrm{H}-\mathrm{O}-\mathrm{H}$ bending and $\mathrm{Si}-\mathrm{O}(\mathrm{Si}),(\mathrm{Al})$ stretching vibrations, respectively. There are also two small absorbing bands at $798 \mathrm{~cm}^{-1}$ and $608 \mathrm{~cm}^{-1}$ which are related to the stretching vibrations of the bridges of $\mathrm{Si}-\mathrm{O}(\mathrm{Si})$ and $\mathrm{Si}-\mathrm{O}$ (Al) groups [24]. In neat hydrogel's FTIR spectrum, it is observed that all peaks belonged to gelatin and SCMC are present but two peaks at $1250 \mathrm{~cm}^{-1}$ and $620 \mathrm{~cm}^{-1}$ which were sharp peaks in SCMC FTIR spectrum, have been weakened severely that confirms the successful graft co-polymerization process. By comparing the FTIR spectra of neat and nano-composite hydrogel samples, it is observed that there are three main peaks in nano-composite hydrogel's FTIR spectrum which are not present in neat hydrogel's FTIR spectrum. These absorbing bands are observed at $1032 \mathrm{~cm}^{-1}, 798 \mathrm{~cm}^{-1}$ and $605 \mathrm{~cm}^{-1}$ which are characteristic peaks of the clinoptilolite and confirm the successful incorporation of clinoptilolite in nano-composite hydrogel's structure. Also, appearance of the characteristic peaks of gelatin and SCMC in both neat and nano-composite hydrogels' FTIR spectra confirms the successful synthesis process.

\subsection{XRD analysis}

For more structural evaluation of the synthesized nanocomposite hydrogels and also to confirm the successful incorporation of clinoptilolite in nano-composite hydrogel structure, XRD analysis was done. As it is seen in Fig. 4, after sulfating the $C M C$, its amorphous structure was changed into a complete crystalline structure which is due to the fact that SCMC is an ionic crystalline solid while CMC is a natural amorphous polysaccharide. The significant difference between XRD patterns of CMC and SCMC, confirms the successful synthesis of the SCMC. Additionally, the XRD pattern of clinoptilolite confirms that it is a crystalline solid and incorporation of it in a hydrogel may affect the hydrogel's structure and may increase its crystallinity. By comparing the XRD patterns of neat and nano-composite hydrogel samples, it is concluded that the incorporation of clinoptilolite has no significant effect on the structure of the synthesized hydrogel samples. This phenomenon is due to the complete dispersion of the clinoptilolite Nanoparticles in the polymeric matrix. As it is seen in Fig. 4, the XRD patterns of the both neat and nano-composite hydrogels are similar but the nano-composite hydrogel sample has sharper peaks which can be due to the presence of clinoptilolite. Furthermore, the intensity of the peak at $2 \theta=22.63^{\circ}$ is the same with its intensity at XRD pattern of clinoptilolite which can confirm the incorporation of clinoptilolite in nano-composite hydrogel's structure. Also, at $2 \theta=3.18^{\circ}$, the intensity of the peak has decreased in comparison to the neat hydrogel that confirms the presence of clinoptilolite in nano-composite hydrogel's structure. Finally, it should be mentioned that by graft copolymerization of the SCMC with poly (AA-CO-AAm) in the presence of gelatin a hydrogel sample with an amorphous structure is obtained in which the crystallinity of the SCMC and clinoptilolite has no significant effect. 


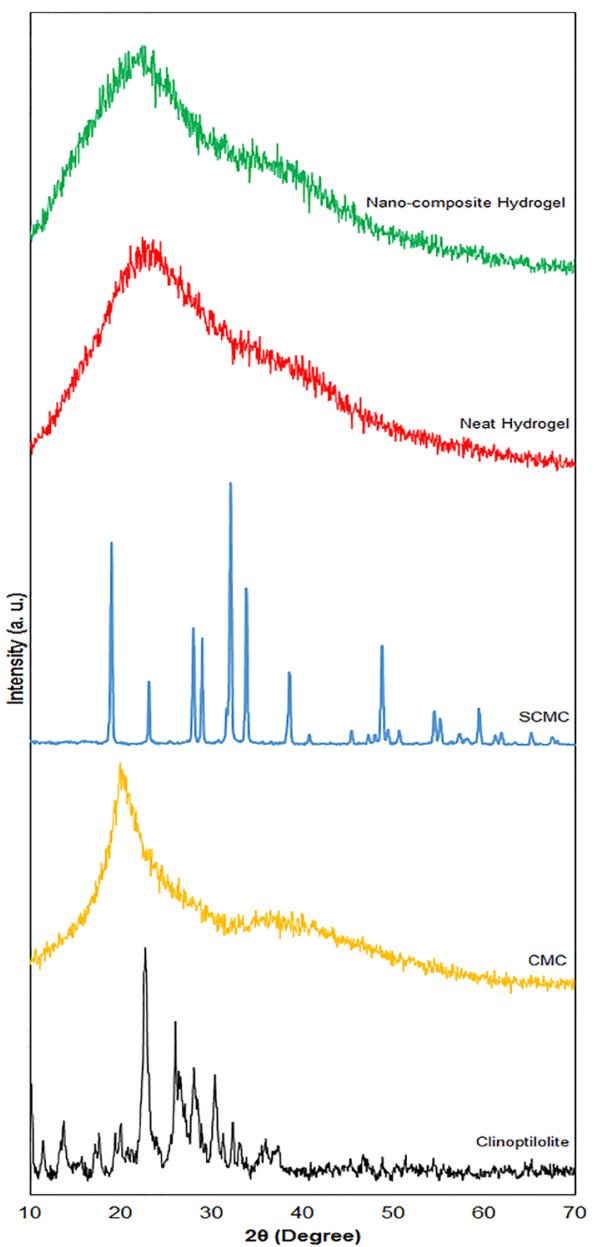

Fig. 4 XRD patterns of grafting natural polymer, additive, neat and nano-composite hydrogel

\subsection{Studying the effect of clinoptilolite addition on water absorption kinetics}

\subsubsection{Equilibrium water absorption}

Figure 5 a shows the equilibrium water absorption graphs for neat hydrogel and nano-composite hydrogel samples with $1 \%, 3 \%, 5 \%, 7 \%$ and $10 \%$ W/W of clinoptilolite with respect to the gelatin quantity. As it is seen in this figure, the equilibrium water absorption amount is the lowest for neat hydrogel which is due to its non-porous structure. For hydrogels with 1\% W/W and $10 \%$ W/W of clinoptilolite, the water uptake amount is less than the other nanocomposite hydrogel samples which may be related to the lack of effective porosity. For nano-composite hydrogel sample with $1 \%$ W/W of clinoptilolite content, the porosity is not as much for a high water absorption amount. Also, for the nano-composite hydrogel sample with $10 \% \mathrm{~W} / \mathrm{W}$ of clinoptilolite content, the high amounts of additive leads to high physical cross linking density and the water uptake amount is decreased which is due to the creation of a compact structure. The equilibrium water absorption amount for rest of the nano-composite hydrogel samples (with 3\% W/W and 7\%W/W of clinoptilolite content) are almost equal but for the sample with $5 \%$ W/W of clinoptilolite content its amount is the mean which shows that this sample can be a good candidate as a nano-composite water reservoir material. The optimum water absorption amount for this sample is due to the optimum porosity and cross linking density in its structure.

\subsubsection{Effect of clinoptilolite on swelling kinetics}

As it was mentioned before, the nano-composite hydrogels samples were synthesized by addition of $1 \%, 3 \%$, $5 \%, 7 \%$ and $10 \% \mathrm{~W} / \mathrm{W}$ of clinoptilolite with respect to the gelatin weight. To investigate the effect of clinoptilolite content on the swelling kinetics of the synthesized nanocomposite hydrogel samples, the t/W versus time graphs for each sample were plotted. By using the obtained graphs which are shown in Fig. 5b, and according to the slope and intercept of the graphs, $\mathrm{k}_{\mathrm{s}}$ and theoretical $\mathrm{W}_{\infty}$ were calculated and the obtained data were collected in Table 3. According to the obtained data, it is concluded that for neat hydrogel which has no porosity in its structure, the swelling rate is high. This phenomenon is related to this fact that for hydrogels with non-porous structure, the water is absorbed only on the surface of the hydrogel so that the swelling rate is high and the hydrogel is got swollen faster. By increasing the porosity in hydrogels, the swelling rate is decreased which is due to the fact that in porous hydrogels more time is needed for water molecules to penetrate into the hydrogels structure [2]. In hydrogels with $1 \%, 3 \%$ and $7 \%$ W/W of clinoptilolite content, the $\mathrm{k}_{\mathrm{s}}$ values are very low which shows that the time period of water penetration into the structure of these hydrogels is too long that confirms the ineffective structural porosity of these hydrogel samples. For the nano-composite hydrogel sample with $10 \% \mathrm{~W} / \mathrm{W}$ of clinoptilolite, the $\mathrm{k}_{\mathrm{s}}$ value is more than the other nano-composite hydrogel samples which shows that the swelling rate is high for this sample. This is due to the high physical cross linking density in this hydrogel sample. Eventually, for the nano-composite hydrogel sample with $5 \% \mathrm{~W} / \mathrm{W}$ of clinoptilolite, the $\mathrm{k}_{\mathrm{s}}$ value is neither very low nor very high which shows that the porosity of this sample is probably optimum.

\subsubsection{Effect of clinoptilolite on water absorption rate and diffusion mechanism}

According to the further information which are obtained from the Table 3, the water absorption rate of each hydrogel sample and also the diffusion mechanism in 

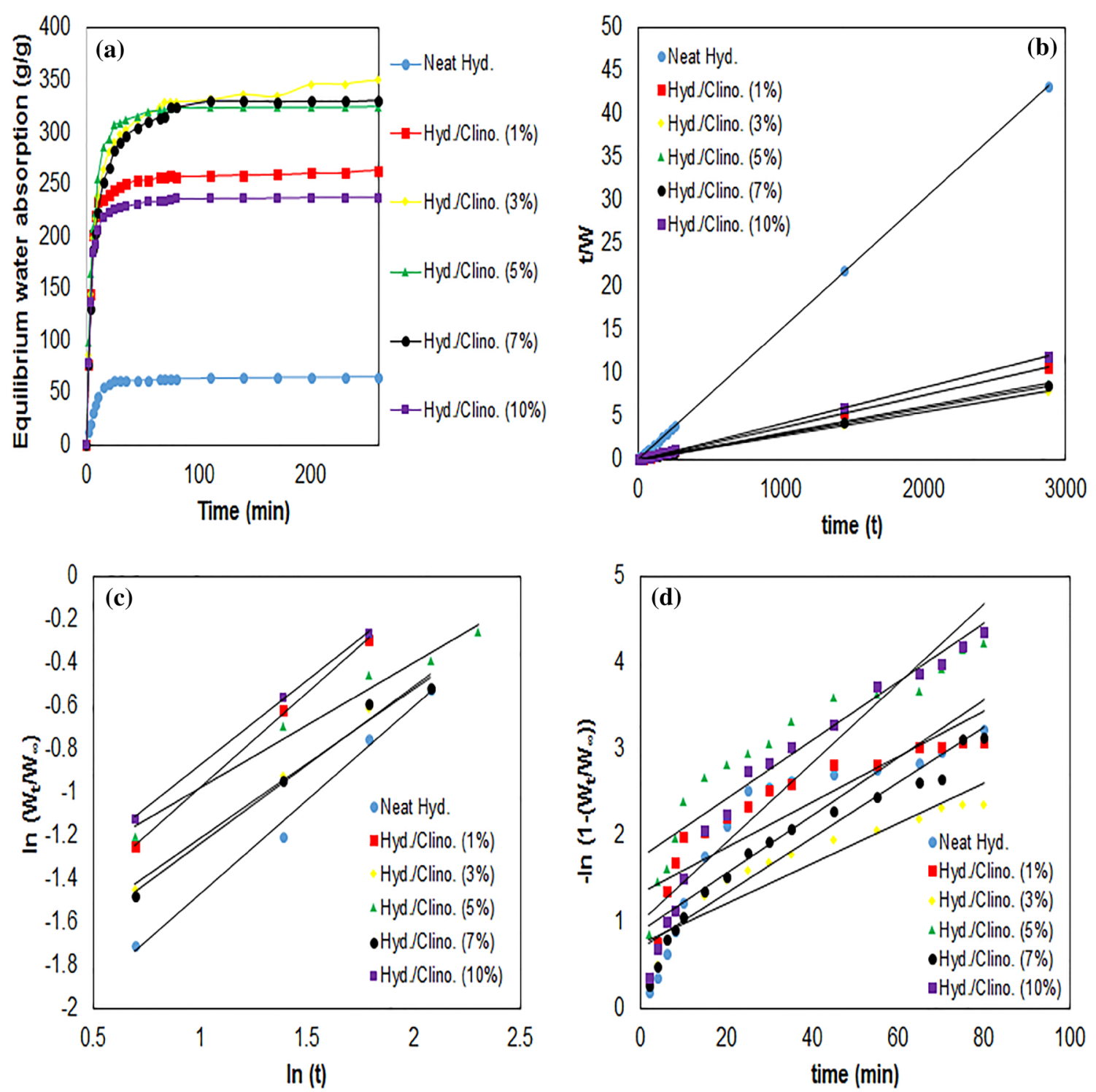

Fig. 5 Equilibrium water uptake (a), swelling rate (b) absorption mechanism (c) and water absorption rate (d) graphs of neat and nanocomposite hydrogels

Table 3 Swelling, diffusion and water uptake parameters of neat ad nano-composite hydrogels

\begin{tabular}{llllllll}
\hline Equation & Parameter & Neat hyd. & $1 \%$ NC hyd. & $3 \%$ NC hyd. & $5 \%$ NC hyd. & $7 \%$ NC hyd. & $10 \%$ NC hyd. \\
\hline$\frac{t}{W}=A+B t$ & $k_{s}$ & $3.2 \times 10^{-3}$ & $9 \times 10^{-4}$ & $4.8 \times 10^{-4}$ & $1.2 \times 10^{-3}$ & $5.6 \times 10^{-4}$ & $2.1 \times 10^{-3}$ \\
& $W_{\infty}$ & 70 & 270.27 & 357.14 & 333.33 & 334 & 238.1 \\
$\frac{W_{t}}{W_{\infty}}=k t^{n}$ & $n$ & 0.866 & 0.875 & 0.691 & 0.583 & 0.726 & 0.783 \\
$W_{t}=W_{\infty}\left(1-e^{-k_{w} t}\right)$ & $k_{w}$ & 0.0335 & 0.0264 & 0.0232 & 0.0462 & 0.0321 & 0.034 \\
\hline
\end{tabular}

each sample can be evaluated. Figure $5 c$, $d$ show the In $\left(\mathrm{Wt} / \mathrm{W}_{\infty}\right)$ versus $\ln (\mathrm{t})$ and $-\ln \left(1-\left(\mathrm{W}_{\mathrm{t}} / \mathrm{W}_{\infty}\right)\right)$ versus time graphs. It is seen that the lowest amounts of $k_{w}$ are belonged to the nano-composite hydrogel samples with $1 \%$ and $3 \% \mathrm{~W} / \mathrm{W}$ of clinoptilolite content. This is due to the creation of ineffective pores in these hydrogels structure in which the water is not able to penetrate into the hydrogel's structure easily. Also, as it is observed in Fig. $5 \mathrm{~d}$ and Table 3, for nano-composite hydrogel samples with $7 \%$ and $10 \% \mathrm{~W} / \mathrm{W}$ of clinoptilolite content, the 
water absorption rates are more than nano-composite hydrogels with $1 \%$ and $3 \% \mathrm{~W} / \mathrm{W}$ of clinoptilolite content which may be due to the better porosity in their structure. For the nano-composite hydrogel with $5 \% \mathrm{~W} / \mathrm{W}$ of clinoptilolite content, the $k_{w}$ value is the highest which confirms that the porosity of this sample is more effective and better than the other samples which is due to the optimum amount of clinoptilolite. Furthermore, for neat hydrogel, the water absorption rate is lower than that of nano-composite hydrogel sample with $5 \% \mathrm{~W} / \mathrm{W}$ of clinoptiloite which is due the lack of optimum porosity. It is necessary to be mentioned that, for neat hydrogel the $\mathrm{k}_{\mathrm{w}}$ value is more than the other nano-composite hydrogel samples except the nano-composite hydrogel sample with $5 \% \mathrm{~W} / \mathrm{W}$ of clinoptilolite content. This phenomenon is due to the fact that in neat hydrogel the water is only interacts with the hydrophilic functional groups on the surface of the hydrogel which leads to easy water absorption on the surface, hence it increases the water absorption rate. But for nano-composite hydrogels which have not optimum porosity, the available hydrophilic functional groups are lower due to the entanglement of the polymer chains in the hydrogels' structure, so the water absorption rate is decreased. To investigate the water absorption mechanism in the synthesized neat and nano-composite hydrogels, the obtained " $n$ " values were studied. As it is seen in Fig. $5 c$ and Table 3, for all of the hydrogel samples the " $n$ " value is more than 0.5 which confirms that the diffusion mechanism is non-Fickian. So, the rate of water diffusion into the hydrogel structure is equal to the relaxation rate of the hydrogels' polymeric network which is a common phenomenon in hydrogels.

\subsection{Effect of clinoptilolite on gel characteristics of the synthesized hydrogels}

To evaluate the effect of addition of clinoptilolite on gel characteristics of the synthesized nano-composite hydrogel samples in comparison with the neat hydrogel, the rheological analysis was accomplished by recording mechanical responses of hydrogel samples within angular frequency range of $0.1-100 \mathrm{~Hz}$. The LVE region for hydrogel samples in which G' (storage modulus) and G" (loss modulus) values are independent of the applied strain, was determined by subjecting the hydrogel samples to strain sweep tests at a constant frequency $(\omega=10 \mathrm{~Hz})$. Figure 6a illustrates that the $\mathrm{G}^{\prime}$ and $\mathrm{G}^{\prime \prime}$ values for each hydrogel sample, are independent of the applied strain below the $0.5 \%$ deformation, which indicates the LVE region. Accordingly, the strain amplitude was determined at $0.5 \%$. Also, the $\mathrm{G}^{\prime}$ and $\mathrm{G}^{\prime \prime}$ values for the synthesized neat and nano-composite hydrogel samples have been depicted in Fig. 6b, as the function of oscillatory frequencies between 0.1 and $100 \mathrm{~Hz}$. Here are two phenomena that determine the gel characteristics of the synthesized hydrogels. First, the position of $G^{\prime}$ graph and second, the loss modulus percentage. The higher a $G^{\prime}$ graph is placed in the $G^{\prime}$ versus $\omega$ diagram, the more a hydrogel sample is stronger and stiffer. Additionally, the
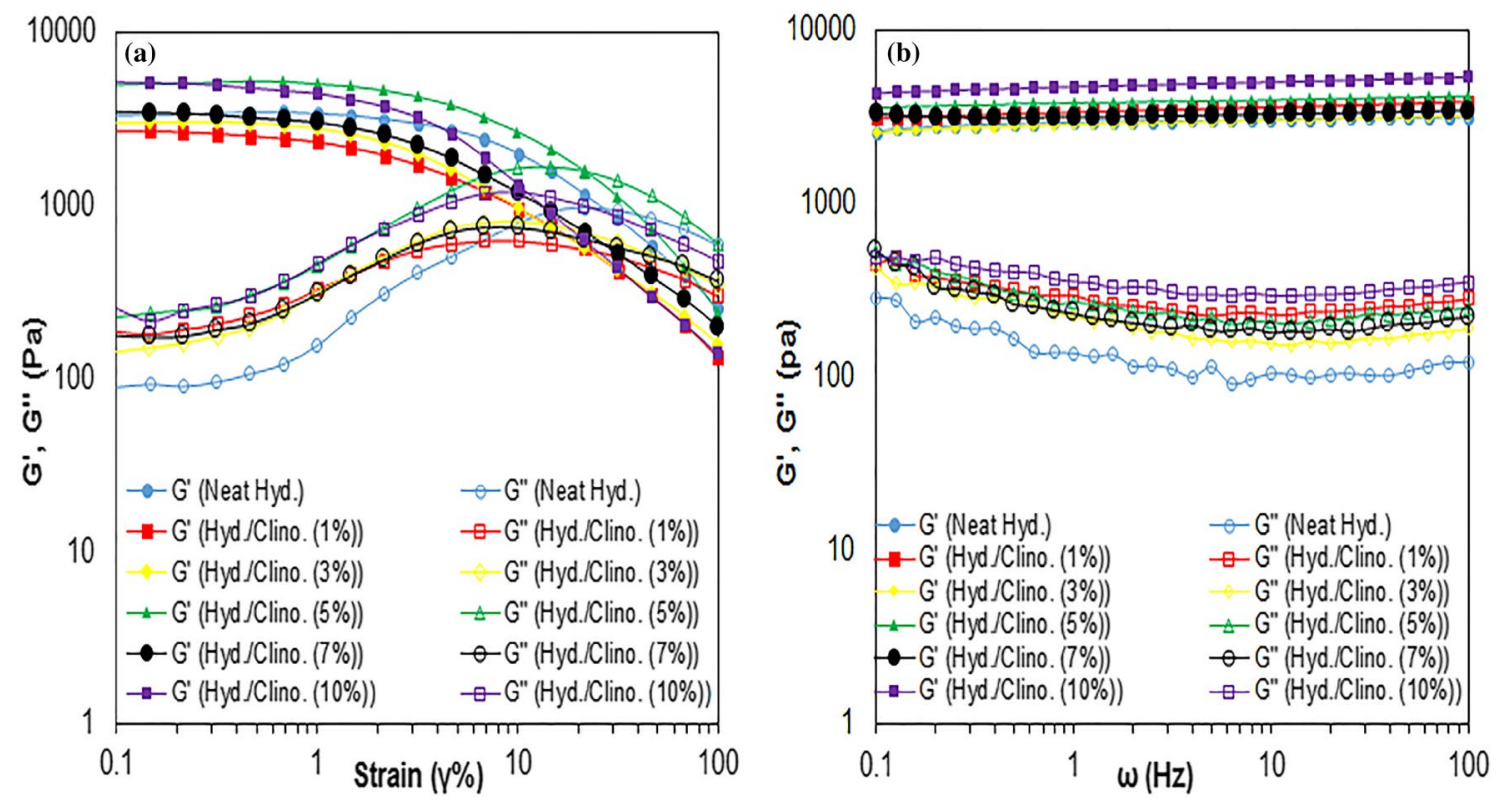

Fig. 6 Strain dependence of the storage modulus ( $\left.G^{\prime}\right)$ and loss modulus ( $\left.G^{\prime \prime}\right)$ at a constant angular frequency $(\omega=10 \mathrm{~Hz})(\mathbf{a})$, and frequency dependence of storage modulus $(\mathrm{G})$ and loss modulus $\left(\mathrm{G}^{\prime \prime}\right)$ at a constant strain $(0.5 \%)$ for neat and nano-composite hydrogel samples (b) 
more loss modulus percentage is, the more a hydrogel is stronger and stiffer. As it can be seen in Fig. 6b, for all hydrogel samples the $G^{\prime}$ values are significantly more than $G^{\prime \prime}$ values at all frequencies which confirms that the elastic response of all hydrogel samples is considerably stronger than their viscose response. Also, as it is seen in this figure for neat and nano-composite hydrogel samples the loss modulus percentages were obtained as $57.58 \%, 37.02 \%, 55.76 \%, 57.97 \%, 52.53 \%$ and $27.84 \%$ for neat hydrogel and nano-composite hydrogels with $1 \%, 3 \%, 5 \%, 7 \%$, and $10 \% \mathrm{~W} / \mathrm{W}$ of clinoptilolite content, respectively. Referring to the $\mathrm{G}^{\prime}$ graphs and loss modulus percentages for synthesized hydrogel samples, it is concluded that the nano-composite hydrogel sample with $5 \% \mathrm{~W} / \mathrm{W}$ of clinoptilolite has the best gel properties among all of the synthesized hydrogel samples. This conclusion is due to the fact that the nano-composite hydrogel sample with $5 \% \mathrm{~W} / \mathrm{W}$ of clinoptilolite content not only has $\mathrm{G}^{\prime}$ values higher than the most of the synthesized hydrogel samples, but also its loss modulus percentage is the highest which both confirm the better gel characteristics of this nano-composite hydrogel sample.

\section{Conclusion}

According to the obtained results, it is concluded that the incorporation of clinoptilolite has a significant effect on water absorption and gel characteristics of the synthesized gelatin/SCMC-g-poly (AA-co-AAm)/clinoptilolite nano-composite hydrogels which is due to the creation of physical cross links which lead to the creation of porous hydrogel structures. Additionally, the obtained results showed that there is an optimum amount for clinoptilolite $(5 \% \mathrm{~W} / \mathrm{W})$ that tangibly affects the structural properties of the synthesized nano-composite hydrogels. Water absorption kinetics studies and rheological evaluations confirmed that the addition of $5 \% \mathrm{~W} / \mathrm{W}$ of clinoptilolite with respect to the gelatin amount has the best effect on structural properties of the hydrogel sample. Eventually, it is necessary to be mentioned that incorporation of 5\% W/W of clinoptilolite leads to the best porosity and also best physical cross linking density which cause to better water absorption properties and gel characteristics. As it is mentioned before, according to the bio compatibility and bio degradability of the polymeric system of the synthesized nano-composite hydrogels, these materials can potentially be used as bio materials and hygienic products. Additionally, according to the water absorption properties and gel characteristics of the obtained nano-composite hydrogels, they can be used in baby diapers or as wound dressing materials.
Acknowledgements The University of Tabriz is gratefully acknowledged for its supports to fulfill this research.

\section{Compliance with ethical standards}

Conflict of interest The authors declare that they have no conflict of interest.

\section{References}

1. Olad A, Zebhi H, Salari D, Mirmohseni A, Reyhanitabar A (2018) Synthesis, characterization, and swelling kinetic study of porous superabsorbent hydrogel nanocomposite based on sulfonated carboxymethylcellulose and silica nanoparticles. J Porous Mater 25:1325-1335

2. Gharekhani $H$, Olad A, Mirmohseni A, Bybordi A (2017) Superabsorbent hydrogel made of NaAlg-g-poly (AA-CO-AAm) and rice husk ash: synthesis, characterization, and swelling kinetic studies. Carbohydr Polym 168:1-13

3. Ilić-Stojanović S, Nikolić L, Nikolić V, Ristić I, Budinski-Simendić J, Kapor A, Nikolić GM (2014) The structure characterization of thermosensitive poly ( $\mathrm{N}$-isopropylacrylamide-co-2-hydroxypropyl methacrylate) hydrogel. Polym Int 63:973-981

4. Olad A, Zebhi H, Salari D, Mirmohseni A, Tabar AR (2018) Water retention and slow release studies of a salep-based hydrogel nanocomposite reinforced with montmorillonite clay. New J Chem 42:2758-2766

5. Rashidzadeh A, Olad A, Salari D, Reyhanitabar A (2014) On the preparation and swelling properties of hydrogel nanocomposite based on sodium alginate-g-poly (acrylic acid-co-acrylamide)/ clinoptilolite and its application as slow release fertilizer. J Polym Res 21:344-359

6. Ni B, Liu M, Lu S, Xie L, Wang Y (2011) Environmentally friendly slow-release nitrogen fertilizer. J Agric Food Chem 59:10169-10175

7. Li L, Smitthipong $W$, Zeng $H$ (2015) Mussel-inspired hydrogels for biomedical and environmental applications. Polym Chem 6:353-358

8. Caló E, Khutoryanskiy VV (2015) Biomedical applications of hydrogels: a review of patents and commercial products. Eur Polym J 65:252-267

9. Albertsson AC, Voepel J, Edlund U, Dahlman O, Soderqvist-Lindblad M (2010) Design of renewable hydrogel release systems from fiberboard mill wastewater. Biomacromol 11:1406-1411

10. Olad A, Doustdar F, Gharekhani H (2018) Starch-based semi-IPN hydrogel nanocomposite integrated with clinoptilolite: preparation and swelling kinetic study. Carbohydr Polym 200:516-528

11. Kosemund K, Schlatter H, Ochsenhirt JL, Krause EL, Marsman DS, Erasala GN (2009) Safety evaluation of superabsorbent baby diapers. Regul Toxicol Pharmacol 53:81-89

12. Zdravković A, Nikolić L, Ilić-Stojanović S, Nikolić V, Najman $S$, Mitić Ž, Ćirić A, Petrović S (2018) The removal of heavy metal ions from aqueous solutions by hydrogels based on $\mathrm{N}$-isopropylacrylamide and acrylic acid. Polym Bull 75:4797-4821

13. Wang X, Strand SP, Du Y, Vårum KM (2010) Chitosan-DNA-rectorite nanocomposites: effect of chitosan chain length and glycosylation. Carbohydr Polym 79:590-596

14. Naahidi S, Jafari M, Logan M, Wang Y, Yuan Y, Bae H, Dixon B, Chen P (2017) Biocompatibility of hydrogel-based scaffolds for tissue engineering applications. Biotechnol Adv 35:530-544

15. Ilić-Stojanović S, Nikolić V, Kundaković T, Savić I, Savić-Gajić I, Jocić E, Nikolić L (2018) Thermosensitive hydrogels for modified 
release of ellagic acid obtained from Alchemilla vulgaris $\mathrm{L}$. extract. Int J Polym Mater Polym Biomater 67:553-563

16. Olad A, Zebhi H, Salari D, Mirmohseni A, Reyhanitabar A (2018) A promising porous polymer-nanoclay hydrogel nanocomposite as water reservoir material: synthesis and kinetic study. J Porous Mater 1:1-11

17. Cong HP, Wang P, Yu SH (2014) Highly elastic and superstretchable graphene oxide/polyacrylamide hydrogels. Small 10:448-453

18. Baek S, Kim B (2003) Synthesis of polyacrylamide/polyurethane hydrogels by latex IPN and AB crosslinked polymers. Colloids Surf A 220:191-198

19. Olad A, Pourkhiyabi M, Gharekhani H, Doustdar F (2018) SemiIPN superabsorbent nanocomposite based on sodium alginate and montmorillonite: reaction parameters and swelling characteristics. Carbohydr Polym 190:295-306

20. Ilić-Stojanović S, Nikolić LB, Nikolić VD, Milić JR, Stamenković J, Nikolić GM, Petrović SD (2013) Synthesis and characterization of thermosensitive hydrogels and the investigation of modifiedrelease of ibuprofen. Hem Ind 67:901-912

21. Kozhunova EY, Makhaeva EE, Khokhlov AR (2012) Collapse of thermosensitive polyelectrolyte semi-interpenetrating networks. Polymer 53:2379-2384

22. Zhang Y, An R, Han L, Wang X, Shi L, Ran R (2018) Novel selfhealing, shape-memory, tunable double-layer actuators based on semi-IPN and physical double-network hydrogels. Macromol Mater Eng 303:1800505-1800513

23. Haraguchi K, Takehisa $T$ (2002) Nanocomposite hydrogels: a unique organic-inorganic network structure with extraordinary mechanical, optical, and swelling/de-swelling properties. Adv Mater 14:1120-1124

24. Olad A, Gharekhani H, Mirmohseni A, Bybordi A (2016) Study on the synergistic effect of clinoptilolite on the swelling kinetic and slow release behavior of maize bran-based superabsorbent nanocomposite. J Polym Res 23:241-255

25. Yu X, Li Y, Yang J, Chen F, Tang Z, Zhu L, Qin G, Dai Y, Chen Q (2018) Nanoclay reinforced self-cross-linking poly (N-hydroxyethyl acrylamide) hydrogels with integrated high performances. Macromol Mater Eng 303:1800295-1800306

26. Hu Y, Du Z, Deng X, Wang T, Yang Z, Zhou W, Wang C (2016) Dual physically cross-linked hydrogels with high stretchability, toughness, and good self-recoverability. Macromolecules 49:5660-5668

27. Olad A, Gharekhani H, Mirmohseni A, Bybordi A (2017) Synthesis, characterization, and fertilizer release study of the salt and $\mathrm{pH}$-sensitive NaAlg-g-poly (AA-co-AAm)/RHA superabsorbent nanocomposite. Polym Bull 74:3353-3377

28. Zdravković AS, Nikolić LB, llić-Stojanović SS, Nikolić VD, Savić SR, Kapor AJ (2017) The evaluation of temperature and pH influences on equilibrium swelling of poly ( $\mathrm{N}$-isopropylacrylamideco-acrylic acid) hydrogels. Hem Ind 71:395-405

29. Masaro L, Zhu X (1999) Physical models of diffusion for polymer solutions, gels and solids. Prog Polym Sci 24:731-775

30. Ferreira JA, Grassi M, Gudiño E, De Oliveira P (2015) A new look to non-Fickian diffusion. Appl Math Model 39:194-204

31. Hossana MJ, Gafurb M, Kadirb M, Karima MM (2014) Preparation and characterization of gelatin-hydroxyapatite composite for bone tissue engineering. Int J Eng Technol 14:24-32

32. Birshtein VY, Tul'chinskii V (1982) A study of gelatin by IR spectroscopy. Chem Nat Compd 18:697-700

Publisher's Note Springer Nature remains neutral with regard to jurisdictional claims in published maps and institutional affiliations. 